\title{
CASE REPORT: ISCHEMIC STROKES AND KINKINGS OF INTERNAL CAROTID ARTERIES IN YOUNG WOMEN. ANTIPHOSPHOLIPID SYNDROME OR TAKAYASU ARTERITIS?
}

Lidiane Moreira Lima Rebouças ${ }^{1, *}$, Alexandra de Oliveira Lopes², Samara Belchior Gaído², Camila Maria Bomtempo Seba de Souza ${ }^{3}$, Maria Carolina Albuquerque de Sousa Martins² ${ }^{2}$ Bruno Guedes Alcoforado Aguiar ${ }^{4}$, Elton Gonçalo de Oliveira Junior ${ }^{1}$, Raquel Moraes da Rocha Nogueira ${ }^{1,3}$

1. Hospital Dr. Carlos Macieira, São Luís (MA), Brazil. 2. Universidade Estadual do Maranhão, São Luís (MA), Brazil. 3. Universidade CEUMA, São Luís (MA), Brazil. 4. Universidade Federal do Piauí, Teresina (PI), Brazil.

*Corresponding author: lidianemIrfisio@gmail.com

\section{BACKGROUND}

Strokes in young people are events usually caused by coagulation disorders, immune, inflammatory diseases or drug use. Among the immunological etiologies, systemic lupus erythematosus (SLE), antiphospholipid syndrome (APS) and Takayasu arteritis are important causes to be considered in young women. This study is a case report of ischemic stroke in a young woman with kinked internal carotid artery.

\section{CASE REPORT}

A 41-year-old female with past medical history of hypertension and deep venous thrombosis (DVT) in the left lower limb at 20 years old was presented with frontal headache, paresthesias of the 3rd and 4th fingers of the right upper limb and monoparesis of the right lower limb. On examination, chronic cutaneous ulcer was found in the left lower limb and a difference in blood pressure between the upper limbs was noted. She denied abortions, fetal losses or prematurity. Laboratory tests: urine summary - proteins 300 mg/dL; antinuclear factor (ANF) 1:640 fine speckled nuclear pattern; lupus anticoagulant 1.37; anticardiolipin IgM 54.4 and IgG > 150; reagent VDRL 1:2; C3 7.7; C4 1.6; erythrocyte sedimentation rate (ESR) 51 mm. Anti-DNA, Anti-Ro, Anti-La and Anti-Sm negative. Magnetic resonance imaging of the brain showed recent and chronic ischemic foci in cerebellar hemispheres, as well as punctate in the frontal, parietal and occipital cortical and subcortical cortico-subcortical centers (Fig. 1). Computed tomography angiography of carotid and vertebral arteries demonstrated kinking of the internal carotid arteries, stenosis of about $50 \%$ of the petrous segment of the right internal carotid artery and focal occlusion of $0.5 \mathrm{~cm}$ left subclavian artery (Fig. 2). Renal biopsy revealed ischemic changes typical of thrombotic microangiopathy and immunofluorescence with deposit of lgG, C3 and C1q in basement membrane (Class V). In light of these findings, the diagnosis of APS secondary to SLE was defined. Because the clinical expression of the disease is strongly related to APS, the main treatment consisted of pulse therapy with methylprednisolone $1 \mathrm{~g} \mathrm{EV}$ for 3 days and full anticoagulation with enoxaparin 1 $\mathrm{mg} / \mathrm{kg}, \mathrm{SC}, 12 / 12 \mathrm{~h}$ with transition to: warfarin (dose adjusted for INR 2-3), prednisone $1 \mathrm{mg} / \mathrm{kg} /$ day and hydroxychloroquine $400 \mathrm{mg} /$ day.

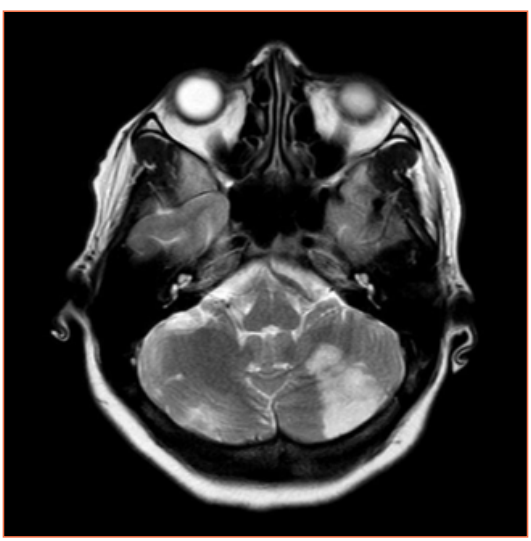

Figure 1. T2-weighted MRI showing ischemic stroke in the left cerebellar. 


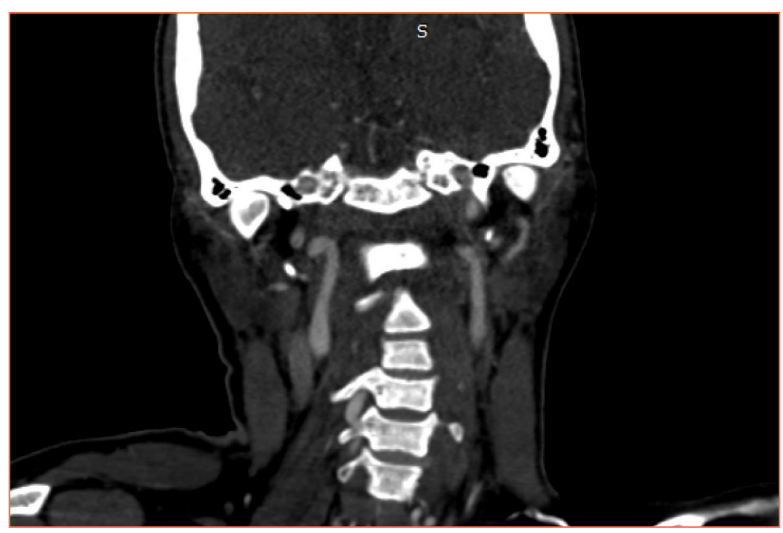

Figure 2. Computed tomography angiography of carotid and vertebral arteries demonstrated kinking of the internal carotid arteries.

\section{CONCLUSION}

Antiphospholipid syndrome, an important cause of recurrent venous and/or arterial thrombosis, presented in this case with DVT, chronic skin ulcer, ischemic stroke and renal thrombotic microangiopathy. Kinked carotid, a condition generally congenital, probably worsened by the activity of rheumatological disease, predisposing to stroke. In this case, the strong clinical expression of APS stands out, with thrombotic vasculopathies and few immunomediated findings, expressed by clinical manifestations and renal biopsy. 